Bull. Korean Math. Soc. 51 (2014), No. 4, pp. 1205-1210

http://dx.doi.org/10.4134/BKMS.2014.51.4.1205

\title{
RELATIVE RELATION MODULES OF FINITE ELEMENTARY ABELIAN $p$-GROUPS
}

\author{
Mohammad Yamin and Poonam Kumar Sharma
}

\begin{abstract}
Let $E$ be a free product of a finite number of cyclic groups, and $S$ a normal subgroup of $\mathrm{E}$ such that $E / S \cong G$ is finite. For a prime $p, \hat{S}=S / S^{\prime} S^{p}$ may be regarded as an $F_{p} G$-module via conjugation in $E$. The aim of this article is to prove that $\hat{S}$ is decomposable into two indecomposable modules for finite elementary abelian $p$-groups $G$.
\end{abstract}

\section{Introduction}

Consider a short exact sequence $1 \rightarrow S \rightarrow E \stackrel{\psi}{\rightarrow} G \rightarrow 1$, where $G$ is a finite group of order $n$ generated by $X=\left\{g_{i}: 1 \leq i \leq d\right\}$. Let $E$ be a free product of cyclic groups $E_{i}$, where $1 \leq i \leq d$. Let $p$ be a (fixed) prime, and $F_{p}$ the field of $p$-elements. If $E$ is a free group, $\hat{S}=S / S^{\prime} S^{p}$, regarded as an $F_{p}$ G-module, is known as a relation module of $G$. In general $\hat{S}$ is called a relative relation module, and it is said to be minimal if it cannot be generated by fewer than $d$ elements.

Gaschutz [1], Gruenberg [2], Kovacs and Stohr [4], Mittal and Passi [5], and others have studied relation modules. Relative relation modules have been studied by Kimmerle [3], Yamin [8, 9], and Sharma and Yamin [7]. As a direct consequence of ([2, Theorem 2.9]), minimal relation modules of $p$-groups are non-projective and indecomposable. Yamin [8] has proved that relative relation modules of $p$-groups are non-projective. Kimmerle [3] has proved that minimal relative relation modules of finite $p$-groups are indecomposable if $\delta=1$. The same result has been proved by Yamin [9] for $\delta=d$. If $G$ is a finite elementary abelian $p$-group, in this article, we prove that $\hat{S}$ is decomposable into two indecomposable modules for $1<\delta<d$.

Throughout this article, concepts related with groups and their representations are used mainly from Robinson [6], often without reference.

Let $G_{i}$ be the cyclic subgroup of $G$ generated by $g_{i}$ of order $n_{i}$ and $E_{i}$ be the cyclic group generated by $e_{i}$ of order $m_{i}$, where $m_{i}=k_{i} n_{i}, 1 \leq i \leq d$. Let

Received October 30, 2013; Revised December 9, 2013.

2010 Mathematics Subject Classification. 20C05, 16D10, 16D70.

Key words and phrases. free groups, free products, $p$-groups, modules, relation modules. 
$p$ be fixed prime and $F_{p}$ the field of p-elements. Let $k_{i}<\infty$ and $p \nmid k_{i}$ if $i \leq \delta$ and $\delta \leq d$, and $k_{i}=\infty$ or $p \mid k_{i}$ if $\delta+1 \leq i \leq d$.

For an $F_{p} G$-module $V$, define $\varphi V$ to be the smallest submodule of $V$ such that $V / \varphi V$ is completely reducible. Equivalently, $\varphi V$ is the intersection of all maximal submodules of $V$. Set $\varphi^{0} V=V, \varphi^{k} V=\varphi\left(\varphi^{k-1} V\right)$. $\left(\left\{\varphi^{k} V\right\}\right.$ is known as the Loewy series of $V$.) From ([8, Prop 2.10]), we have an $F_{p} G$ exact sequence:

$$
1 \rightarrow \hat{S} \rightarrow \oplus_{i=1}^{\delta} \underline{g}_{i}^{G} \oplus_{i=\delta+1}^{d} b_{i} F_{p} G \stackrel{\hat{\psi}}{\rightarrow} \underline{g} \rightarrow 1,
$$

where $\underline{g}$, which is same as $\varphi F_{p} G$, denotes the augmentation ideal of $F_{p} G$ and $\underline{g}_{i}^{G}$ that of $b_{i} F_{p} G_{i}$ induced to $G$. Moreover $\hat{\psi}$ is determined by $b_{i}\left(1-g_{i}\right) \rightarrow 1-g_{i}$ if $1 \leq i \leq \delta$, and $b_{i} \rightarrow 1-g_{i}$ if $1+\delta \leq i \leq d$.

\section{Structure of relative relation modules}

Theorem 2.1. If $G$ is a finite elementary abelian $p$-group and $1<\delta<d$, then $\hat{S}$ is decomposable into two indecomposable modules.

In order to prove the theorem, we shall construct a minimal generating set for $\hat{S}$. For, we need certain bases for the Loewy factors of $b_{i} F_{p} G$ and $\underline{g}_{i}^{G}$.

Let

Let

$$
B_{i}=\left\{\begin{array}{cl}
g_{i}^{G} & \text { for } 1 \leq i \leq \delta, \\
b_{i} F_{p} G & \text { otherwise; }
\end{array} \text { and } B=\oplus_{i=1}^{d} B_{i}\right.
$$

$$
Y=\left\{y: y \in b_{i} F_{p} G ; y=\prod_{i=1}^{d}\left(1-g_{i}\right)^{\mu_{i}},\left(1-g_{i}\right)^{0}=1, g_{i} \in X, 0 \leq \mu_{i} \leq n_{i}-1\right\}
$$

Clearly, $Y$ is a set of non-zero and distinct elements of $F_{p} G$ and $|Y|=|G|$. Then it can be checked that $Y$ is an $F_{p}$ basis of $F_{p} G$. Now, for $y \in Y$, define $\sum_{i=1}^{d} \mu_{i}$ as the length of $y$. It is easy to observe that the length of $y$ varies from 0 to $l=d(p-1)$. Corresponding to each $k$, let $Y_{k}$ be the set of all elements of $Y$ of length $k$. Then it is easy to ckeck that $Y_{k}$ is a minimal generating set of $\varphi^{k}\left(F_{p} G\right)$, and $\left\{\mathrm{y}+\varphi^{k+1} F_{p} G: y \in Y_{k}\right\}$ is an $F_{p}$ basis of $\varphi^{k}\left(F_{p} G\right) / \varphi^{k+1}\left(F_{p} G\right)$.

For a fixed $i, 1 \leq i \leq \delta$, let $Z^{i}=\left\{z \mid z=b_{i}\left(1-g_{i}\right) y, y \in Y\right.$ and $\left.\left(1-g_{i}\right) y \neq 0\right\}$. Clearly, $\left(1-g_{i}\right) y=0$ if and only if $y=\left(1-g_{i}\right)^{p-1} y^{\prime}$ for some $y^{\prime} \in Y$. The number of such $y$ is exactly $p^{n-1}$. Therefore, $\left|Z^{i}\right|=p^{n}-p^{n-1}=p^{n-1}(p-1)=$ $\operatorname{dim}\left(B_{i}\right)$. In fact $Z^{i}$ is an $F_{p}$ basis of $B_{i}$, and $Z_{k}^{i}$, the set of all elements of $Z^{i}$ of length $k$, is a minimal generating set of $\varphi^{k-1} B_{i}$. Similarly, for a fixed $i$, $\delta+1 \leq i \leq d$, the set $Z^{i}=\left\{b_{i} y, y \in Y\right\}$ is an $F_{p}$ basis of $b_{i} F_{p} G$ and $Z_{k}^{i}$ is a minimal generating set of $\varphi^{k} B_{i}$.

Let $Z=\cup_{i=1}^{d} Z^{i}$ and $Z_{k+1}=\cup_{i=1}^{d} Z_{k+1}^{i}, 0 \leq k \leq l-1$. Then it is easy to see that $Z$ is an $F_{p}$ basis of $B$ and $Z_{k+1}$ is a minimal generating set of $\oplus_{i=1}^{\delta} \varphi^{k} B_{i} \oplus_{i=\delta+1}^{d} \varphi^{k+1} B_{i}$. 
Remark. The Loewy length of $B_{i}= \begin{cases}l & \text { for } 1 \leq i \leq \delta \\ l+1 & \text { for } 1+\delta \leq i \leq d .\end{cases}$

Lemma 2.2. Let $X_{1}=\left\{\left(b_{i}-b_{j}\right)\left(1-g_{i}\right)\left(1-g_{j}\right), 1 \leq i<j \leq \delta\right\}$ and $X_{2}=$ $\left\{b_{i}\left(1-g_{j}\right)-b_{j}\left(1-g_{i}\right), b_{i}\left(1-g_{i}\right)^{n_{i}-1}, 1+\delta \leq i<j \leq d\right\}$. Then $X_{1} \cup X_{2}$ is a minimal generating set of $\hat{S}$.

Proof. Firstly, we note that $\left|X_{1} \cup X_{2}\right|=\frac{1}{2} \delta(\delta-1)+\frac{1}{2}(d-\delta)(d-\delta+1)$. Now we show that $X_{1} \cup X_{2}$ generate $\hat{S}$. If $V$ is any submodule generated by $X_{1} \cup X_{2}$, then $V \subseteq \hat{S}$. For the other inclusion, since $\oplus_{i=1}^{\delta} \varphi^{k} B_{i} \oplus_{i=\delta+1}^{d} \varphi^{k+1} B_{i}=\{0\}$, when $k=l$, therefore it is sufficient to show that an arbitrary element of $\hat{S}$ can be expresses as a sum of an element of $V$ and an element of $\oplus_{i=1}^{\delta} \varphi^{k} B_{i} \oplus_{i=\delta+1}^{d}$ $\varphi^{k+1} B_{i}$ for all $k, 0 \leq k \leq l$. We shall show this by induction on $k$. The result is obviously true for $k=0$. Let $x \in \hat{S}$, and for a fixed $k, 0 \leq k \leq l-1$, suppose that $x=v+x^{\prime}, v \in V$ and $x^{\prime} \in \oplus_{i=1}^{\delta} \varphi^{k} B_{i} \oplus_{i=\delta+1}^{d} \varphi^{k+1} B_{i}$. To complete the induction argument, we shall show that $x^{\prime}=x_{0}+x_{1}$ for some $x_{0} \in V$ and $x_{1} \in \oplus_{i=1}^{\delta} \varphi^{k+1} B_{i} \oplus_{i=\delta+1}^{d} \varphi^{k+2} B_{i}$.

Writing $x^{\prime}$ as a linear combination of an element of $Z_{k+1}$ and then rewritting each element of $F_{p} G$ as a sum of an element of $F_{p}$ and an element of $\underline{g}$, we have

$$
\begin{aligned}
x^{\prime}= & \sum a_{\lambda_{1}} b_{1}\left(1-g_{1}\right) y_{\lambda_{1}}+\cdots+\sum a_{\lambda_{\delta}} b_{\delta}\left(1-g_{\delta}\right) y_{\lambda_{\delta}} \\
& +\sum a_{\lambda_{\delta+1}} b_{\delta+1} y_{\lambda_{\delta+1}}^{\prime}+\cdots+\sum a_{\lambda_{d}} b_{d} y_{\lambda_{d}}^{\prime}+x_{1},
\end{aligned}
$$

where $a_{\lambda_{i}} \in F_{p}, y_{\lambda_{i}} \in Y_{k}, y_{j}^{\prime} \in Y_{k+1}$ and $x_{1} \in \oplus_{i=1}^{\delta} \varphi^{k+1} B_{i} \oplus_{i=\delta+1}^{d} \varphi^{k+2} B_{i}$. Clearly, $\left(1-g_{i}\right) y_{\lambda_{i}} \in Y_{k+1}$, however they need not be distinct if $x_{\lambda}=(1-$ $\left.g_{i}\right) y_{\lambda_{i}}=\left(1-g_{j}\right) y_{\lambda_{j}}=\cdots=\left(1-g_{r}\right) y_{\lambda_{r}}=\left(1-g_{s}\right) y_{\lambda_{s}}=y_{\lambda_{k}}^{\prime}=y_{\lambda_{l}}^{\prime}=y_{\lambda_{m}}^{\prime}=y_{\lambda_{n}}^{\prime}$, where $0 \leq i, j, \ldots, r, s \leq \delta$ and $\delta+1 \leq k, l, \ldots, m, n \leq d$. Let

$$
\begin{aligned}
\mu_{\lambda}= & a_{\lambda_{i}} b_{i}+a_{\lambda_{j}} b_{j}+\cdots+a_{\lambda_{r}} b_{r}+a_{\lambda_{s}} b_{s}+a_{\lambda_{k}} b_{k}+a_{\lambda_{l}} b_{l} \\
& +\cdots+a_{\lambda_{m}} b_{m}+a_{\lambda_{n}} b_{n} .
\end{aligned}
$$

Then $x_{0}=\sum_{\lambda} \mu_{\lambda} x_{\lambda}$, where $x_{\lambda}$ are distinct elements of $Y_{k+1}$. Therefore we have

$$
\begin{aligned}
& x_{0} \hat{\psi}= {\left[\sum _ { \lambda } \left\{\left(a_{\lambda_{i}} b_{i}+a_{\lambda_{j}} b_{j}+\cdots+a_{\lambda_{r}} b_{r}+a_{\lambda_{s}} b_{s}+a_{\lambda_{k}} b_{k}+a_{\lambda_{l}} b_{l}\right.\right.\right.} \\
&\left.\left.\left.+\cdots+a_{\lambda_{m}} b_{m}+a_{\lambda_{n}} b_{n}\right)\right\} x_{\lambda}\right] \hat{\psi} \\
&=\sum_{\lambda}\left\{\left(a_{\lambda_{i}} b_{i}+a_{\lambda_{j}} b_{j}+\cdots+a_{\lambda_{r}} b_{r}+a_{\lambda_{s}} b_{s}\right)+a_{\lambda_{k}}\left(1-g_{k}\right)+a_{\lambda_{l}}\left(1-g_{l}\right)\right. \\
&\left.\quad+\cdots+a_{\lambda_{m}}\left(1-g_{m}\right)+a_{\lambda_{n}}\left(1-g_{n}\right)\right\} x_{\lambda} .
\end{aligned}
$$

Now for $\delta+1 \leq k, l, \ldots, m, n \leq d$, we have $\left(1-g_{k}\right) y_{\lambda_{k}}^{\prime}=\left(1-g_{l}\right) y_{\lambda_{l}}^{\prime}$ if and only if $y_{\lambda_{k}}^{\prime}=\left(1-g_{l}\right) y$ and $y_{\lambda_{l}}^{\prime}=\left(1-g_{k}\right) y$ for some $y \in Y_{k+1}$. Let $x_{\lambda^{\prime}}=\left(1-g_{k}\right) y_{\lambda_{k}}^{\prime}=\left(1-g_{l}\right) y_{\lambda_{l}}^{\prime}=\cdots=\left(1-g_{m}\right) y_{\lambda_{m}}^{\prime}=\left(1-g_{n}\right) y_{\lambda_{n}}^{\prime}$, where the 
$x_{\lambda^{\prime}}$ are distinct elements of $Y_{k+1}$. Then we have

$$
\begin{aligned}
x_{0} \hat{\psi}= & \sum_{\lambda}\left(a_{\lambda_{i}}+a_{\lambda_{j}}+\cdots+a_{\lambda_{r}}+a_{\lambda_{s}}\right) x_{\lambda} \\
& +\sum_{\lambda^{\prime}}\left(a_{\lambda_{k}}+a_{\lambda_{l}}+\cdots+a_{\lambda_{m}}+a_{\lambda_{n}}\right) x_{\lambda^{\prime}} \\
= & \sum_{\lambda} \beta_{\lambda} x_{\lambda}+\sum_{\lambda^{\prime}} \beta_{\lambda^{\prime}} x_{\lambda^{\prime}},
\end{aligned}
$$

where $\beta_{\lambda}=\alpha_{\lambda_{i}}+\alpha_{\lambda_{j}}+\cdots+\alpha_{\lambda_{r}}+\alpha_{\lambda_{s}}$ and $\beta_{\lambda^{\prime}}=\alpha_{\lambda_{k}}+\alpha_{\lambda_{l}}+\cdots+\alpha_{\lambda_{m}}+$ $\alpha_{\lambda_{n}}$. Clearly, $0=x \hat{\psi}=v \hat{\psi}+x^{\prime} \hat{\psi}=x^{\prime} \hat{\psi}=x_{0} \hat{\psi}+x_{1} \hat{\psi}$, where $x_{1} \hat{\psi} \in$ $\oplus_{i=1}^{\delta} \varphi^{k+1} B_{i} \oplus_{i=\delta+1}^{d} \varphi^{k+2} B_{i}$.

Now $\left(\oplus_{i=1}^{d} \varphi^{k+1} B_{i}\right) \hat{\psi} \subseteq \varphi^{k+1} \underline{g}$ and $\left(\oplus_{i=1}^{d} \varphi^{k+2} B_{i}\right) \hat{\psi} \subseteq \varphi^{k+2} \underline{g}$. Moreover,

$$
\varphi^{k+2} \underline{g} \subseteq \varphi^{k+1} \underline{g} \text { implies that }\left(\oplus_{i=1}^{\delta} \varphi^{k+1} B_{i} \oplus_{i=\delta+1}^{d} \varphi^{k+2} B_{i}\right) \subseteq \varphi^{k+1} \underline{g} .
$$

But $\varphi^{k+1} \underline{g}=\left(x_{0} \hat{\psi}+x_{1} \hat{\psi}\right)+\varphi^{k+1} \underline{g}=x_{0} \hat{\psi}+\varphi^{k+1} \underline{g}=\sum \beta_{\lambda} x_{\lambda}+\sum \beta_{\lambda^{\prime}} x_{\lambda^{\prime}}+\varphi^{k+1} \underline{g}$. Since each $x_{\lambda}$ and $x_{\lambda^{\prime}}$ are distinct elements of $Y_{k+1}$ and $Y_{k+2}$, respectively, therefore, each $\beta_{\lambda}=\beta_{\lambda^{\prime}}=0$. Therefore, $\alpha_{\lambda_{s}}=-\alpha_{\lambda_{i}}-\alpha_{\lambda_{j}}-\cdots-\alpha_{\lambda_{r}}$ and $\alpha_{\lambda_{n}}=-\alpha_{\lambda_{k}}-\alpha_{\lambda_{l}}-\cdots-\alpha_{\lambda_{m}}$. Thus

$$
\begin{aligned}
x_{0}=\sum_{\lambda}\left\{a_{\lambda_{i}} b_{i}+a_{\lambda_{j}} b_{j}+\cdots+a_{\lambda_{r}} b_{r}-\left(a_{\lambda_{i}}+a_{\lambda_{j}}+\cdots+a_{\lambda_{r}}\right) b_{s}\right\} x_{\lambda} \\
\quad+\left\{a_{\lambda_{k}} b_{k}+a_{\lambda_{l}} b_{l}+\cdots+a_{\lambda_{m}} b_{m}-\left(a_{\lambda_{k}}+a_{\lambda_{l}}+\cdots+a_{\lambda_{m}}\right) b_{n}\right\} x_{\lambda} \\
=\sum_{\lambda}\left\{a_{\lambda_{i}}\left(b_{i}-b_{s}\right)+a_{\lambda_{j}}\left(b_{j}-b_{s}\right)+\cdots+a_{\lambda_{r}}\left(b_{r}-b_{s}\right)\right\} x_{\lambda} \\
+\left\{a_{\lambda_{k}}\left(b_{k} y_{\lambda_{n}}^{\prime}-b_{n} y_{\lambda_{k}}^{\prime}\right)+a_{\lambda_{l}}\left(b_{l} y_{\lambda_{n}}^{\prime}-b_{n} y_{\lambda_{l}}^{\prime}\right)+\cdots\right. \\
\left.\quad+a_{\lambda_{m}}\left(b_{m} y_{\lambda_{n}}^{\prime}-b_{n} y_{\lambda_{m}}^{\prime}\right)\right\}
\end{aligned}
$$

which is an element of $V$. Therefore, $\hat{S} \subseteq V$. Since $X_{1}$ and $X_{2}$ lie in different direct summands of the middle term $\oplus_{i=1}^{\delta} \underline{g}_{i}^{G} \oplus_{i=\delta+1}^{d} b_{i} F_{p} G$ of (1.1), therefore $X_{1} \cap X_{2}=0$. Thus in order to show that $X_{1} \cup X_{2}$ is a minimal generating set of $\hat{S}$, we only need to show that $X_{1}$ and $X_{2}$ are minimal sets of $U$ and $V$, respectively. First we shall show that $X_{1}$ is a minimal generating set for $U$. For, since we are dealing with a finite elementary $p$-group case, it is sufficient to show that no proper subset of $X_{1}$ generates $U$. For, on the contrary suppose that $X_{1}$ is not minimal, and let $\left(b_{\mu}-b_{\nu}\right)\left(1-g_{\mu}\right)\left(1-g_{\nu}\right)=\sum_{(\mu, \nu) \neq(i, j), 1 \leq i<j \leq \delta}\left(b_{i}-\right.$ $\left.b_{j}\right)\left(1-g_{i}\right)\left(1-g_{j}\right) a_{i j}=x$, say, $a_{i j} \in F_{p} G$. Then $b_{\mu}\left(1-g_{\mu}\right)\left(1-g_{\nu}\right)=x+b_{\nu}(1-$ $\left.g_{\mu}\right)\left(1-g_{\nu}\right)$, which is a contradiction because $\cup_{i=1}^{\delta} Z_{2}^{i}$ is a minimal generating set of $\oplus_{i=1}^{\delta} \varphi^{i} B$. This shows that $X_{1}$ is minimal. For proving that $X_{2}$ is a minimal generating set, for convenient reference, we shall call the elements $b_{i}\left(1-g_{j}\right)-b_{j}\left(1-g_{i}\right)$ of type-I and $b_{i}\left(1-g_{i}\right)^{n_{i}-1}$ of type-II. Then it is clear that type-I elements cannot be generated from type-II elements and vice-versa. Suppose on the contrary that the set $X_{2}$ is not minimal and let $b_{\mu}\left(1-g_{\nu}\right)-b_{\nu}(1-$ 
$\left.g_{\mu}\right)=\sum_{(\mu, \nu) \neq(i, j), \delta+1 \leq i<j \leq d}\left\{b_{i}\left(1-g_{j}\right)-b_{j}\left(1-g_{i}\right)\right\} a_{i j}=y$, say, $a_{i j} \in F_{p} G$. Then $b_{\mu}\left(1-g_{\nu}\right)=y+b_{\nu}\left(1-g_{\mu}\right)$, which is a contradiction, because an element of $Z_{1}$ can not be expressed as a linear combination of the remaining elements of $Z_{1}$.

Proposition 2.3. If $U$ and $V$ are $F_{p} G$ modules generated by $X_{1}$ and $X_{2}$, respectively, then $\hat{S} \cong U \oplus V$.

Proof. By Lemma 2.2, it follows that $\hat{S}=U+V$. Moreover, since $X_{1}$ and $X_{2}$ lie in different direct summands of (1.1), $U \cap V=\{0\}$, and hence $\hat{S} \cong U \oplus V$.

In view of Proposition 2.3, to complete the proof of Theorem 2.1, we only need to prove that $U$ and $V$ are indecomposable. For, consider a set

$$
C=\left\{c_{i j}: c_{i j}=\left(b_{i}-b_{j}\right) \prod_{\mu=1}^{d}\left(1-g_{\mu}\right)^{n_{\mu}-1}, 1 \leq i<j \leq \delta\right\} .
$$

Clearly $|C|=\frac{1}{2} \delta(\delta-1)$. Let $U_{1}$ be a submodule of $U$ such that $\operatorname{dim}\left(\left(U_{1}+\right.\right.$ $\varphi U) / \varphi U)=r$, where $0 \leq r \leq \frac{1}{2} \delta(\delta-1)$. Now we shall prove that $U_{1}$ contains at least $\mathrm{r}$ elements of $C$. For, $r=0$, there is nothing to prove. Therefore, suppose that $r>0$ and choose $\left\{u_{m}: u_{m} \in U_{1}, 1 \leq m \leq r\right\}$ such that $\left\{u_{m}+\right.$ $\varphi U, 1 \leq m \leq r\}$ is an $F_{p}$ basis of $\left(U_{1}+\varphi U\right) / \varphi U$. Then

$$
u_{m}=\sum_{1 \leq i<j \leq \delta} \alpha_{m_{i j}}\left(b_{i}-b_{j}\right)\left(1-g_{i}\right)\left(1-g_{j}\right)+w,
$$

where $\alpha_{m_{i j}} \in F_{p}$ and $w \in \varphi U$. Clearly, in the expression of $u_{m}$, at least one $\alpha_{m_{i j}}$ is non-zero. Let $y=\prod_{\lambda=1}^{d}\left(1-g_{\lambda}\right)^{\nu_{\lambda}-1}$, where $\nu_{\lambda}= \begin{cases}n_{\lambda}-2, & \text { if } \lambda=i \text { or } j ; \\ n_{\lambda}-1, & \text { otherwise. }\end{cases}$

Then $u_{m} y=\alpha_{m_{i j}}\left(b_{i}-b_{j}\right) \prod_{\lambda=1}^{d}\left(1-g_{\lambda}\right)^{n_{\lambda}-1}(w y=0)$. So, we get $u_{m} y=$ $\alpha_{m_{i j}} c_{i j}$, and so $\left(\alpha_{m_{i j}}\right)^{-1} u_{m} y=c_{i j} \in U_{1}$. Since at least $r$ of the $\left(b_{i}-b_{j}\right)(1-$ $\left.g_{i}\right)\left(1-g_{j}\right)$ are distinct, $U_{1}$ contains at least $\mathrm{r}$ elements $c_{i j}$ of $C$.

Suppose $U=U_{1} \oplus U_{2}$ and $\operatorname{dim}\left(U_{i} / \varphi U_{i}\right)=\frac{1}{2} \delta(\delta-1)=r_{1}+r_{2}$. From above argument, we infer that $U_{i}$ contains a set $C_{i}$ of at least $r_{i}$ elements of $C$. In fact $C=C_{1} \cup C_{2}$ and $C_{1} \cap C_{2}=\Phi$.

Clearly, $c_{i \delta}=c_{i k}+c_{k \delta}$ for some $1 \leq i \leq \delta-1$ and $1<k<\delta$. Therefore $C$ is an $F_{p}$-linear combination of $\left\{c_{i \delta}: 1 \leq \bar{i} \leq \delta-1\right\}$. Then it is easy to check that either $C \subseteq U_{1}$ or $C \subseteq U_{2}$, and therefore, $U$ is indecomposable.

Now we prove that $V$ is indecomposable. If $d-\delta=1$, then $X_{2}$ contains only one element and therefore $V$ is indecomposable. Now we suppose that $d-\delta \geq 2$ and consider the set $C^{\prime}=\left\{b_{i} \prod_{\mu=1}^{d}\left(1-g_{\mu}\right)^{n_{\mu}-1}: 1+\delta \leq i \leq d\right\}$. (Note that $\left|C^{\prime}\right|=d-\delta$.)

Suppose that $V=V_{1}+V_{2}$. As before, we shall identify the elements $b_{i}(1-$ $\left.g_{j}\right)-b_{j}\left(1-g_{i}\right)$ of $X_{2}$ as those of type-I and $b_{i}\left(1-g_{i}\right)^{n_{i}-1}$ of type-II. Then the following possibilites arise: 
(i) $V_{1}$ contains all elements of $X_{2}$ of type-I and $V_{2}$ contains all elements of $X_{2}$ of type-II.

(ii) $V_{1}$ contains $r_{1}$ elements of type-I and $s_{1}$ elements of type-II, and $V_{2}$ contains $r_{2}$ elements of type-I and $s_{2}$ elements of type-II, where $r_{1}+r_{2}$ $=\frac{1}{2}(d-\delta)(d-\delta-1)$ and $s_{1}+s_{2}=d-\delta$

(iii) $V_{1}$ or $V_{2}$ contain all elements of $X_{2}$.

We shall establish that only (iii) is true by eliminating the other two possibilities. If (i) was true, then both $V_{1}$ and $V_{2}$ would contain all elements of $C^{\prime}$, which would be a contradiction because $V_{1} \cap V_{2}=\{0\}$. Similarly if (ii) was true, then the number of elements in both $V_{1}$ and $V_{2}$ would at least be $\frac{1}{2}(d-\delta)(d-\delta-1)>(d-\delta)$, for $(d-\delta) \geq 2$, which would force $V_{1}$ and $V_{2}$ to contain some common elements of $C^{\prime}$.

Thus only (iii) is true which implies that either $V=V_{1}$ or $V=V_{2}$, and so $V$ is indecomposable, which completes our proof.

\section{References}

[1] W. Gaschutz, Über modulare Darstellungen endlicher Gruppen, die von freien Gruppen induziert warden, Math. Z. 60 (1954), 274-286.

[2] K. W. Gruenberg, Relation modules of finite groups, Regional Conference Series in Math, Number 25, A.M.S. Providence, R.I., 1976.

[3] W. Kimmerle, Relative relation modules on generators for integral group rings of finite groups, Math. Z. 172 (1980), 143-156.

[4] L. G. Kovacs and R. Stohr, Lie powers of relation modules for groups, J. Algebra 326 (2011), no. 1, 192-200.

[5] J. N. Mittal and I. B. S. Passi, Annihilators of relation modules, J. Austral. Math. Soc. (2) 16 (1973), 228-233.

[6] D. J. S. Robinson, A Course in the Theory of Groups, Second Edition. Graduate Texts in Mathematics, 80. Springer-Verlag, New York, 1996.

[7] P. K. Sharma and M. Yamin, Relative Relation Modules of $S L(2, p)$ and $P S L(2, p)$ groups, J. Indian Math Soc. 80 (2013), no. 3-4, 341-348.

[8] M. Yamin, Relative relation modules of finite groups, Proc. Edinburgh Math. Soc. 34 (1991), no. 3, 433-442.

[9] _ Minimal relative relation modules of finite p-groups, Proc. Amer. Math. Soc. 118 (1993), no. 1, 1-3.

MOHAMMAD YAMin

King AbDulaziz University

Jeddah, Saudi Arabia

E-mail address: myamin@kau.edu.sa

Poonam Kumar Sharma

D.A.V. College

JALANDhAR (PunJaB), IndiA

E-mail address: pksharma@davjalandhar.com 